\title{
The urinary catheter technique: A novel approach to right heart catheterization via the antecubital venous system
}

\author{
Michele A. Coceani \\ Fondazione Toscana G. Monasterio, Pisa, Italy. \\ Correspondence: Michele A. Coceani. Address: Via Moruzzi 1, 56124 Pisa, Italy. Email: michecoc@ftgm.it \\ Received: September 4, 2014 \\ Accepted: October 10, $2014 \quad$ Online Published: October 29, 2014 \\ DOI : $10.5430 /$ ijdi.v2n1p20 \\ URL: http://dx.doi.org/10.5430/ijdi.v2n1p20
}

\begin{abstract}
Right heart catheterization permits assessment of intracardiac hemodynamics in various forms of cardiac disease. Although usually performed via a central vein, an antecubital vein may also be accessed. The basilic vein presents the theoretical advantage of draining directly into the subclavian vein, whereas the angle between the cephalic and subclavian veins tends to be rather acute and, consequently, advancement of the Swan-Ganz catheter in this setting may be difficult. In the present report, the Author describes a novel technique which overcomes the tortuosity that is encountered when the Swan-Ganz catheter is advanced from the cephalic vein into the subclavian vein.
\end{abstract}

\section{Keywords}

Diagnostic catheterization, Right heart catheterization, Vascular complications

\section{I ntroduction}

The modern interventional cardiology era has been revolutionized by the introduction of balloons, stents and, more recently, by various devices for structural cardiac disease. Amidst this technological explosion, right heart catheterization (RHC) - the first percutaneous intervention performed in humans in 1929 by Werner Forssmann - has been largely overshadowed. Nevertheless, RHC maintains a central role in the management of various cardiac and pulmonary diseases, such as cardiomyopathies, constrictive pericarditis and pulmonary arterial hypertension. RHC is usually performed via a central vein, which may be femoral, jugular or subclavian. However, central vein access is not exempt from complications, such as hematoma and accidental arterial puncture, which may in turn lead to pseudoaneurysm and arteriovenous fistula ${ }^{[1]}$. For this reason, antecubital veins represent an attractive alternative for access during $\mathrm{RHC}^{[2,3]}$. In the present report, the Author presents a technique which facilitates advancement of the Swan-Ganz catheter for RHC through a cephalic vein.

\section{Case presentation}

A 73-year-old man, with a history of chronic obstructive pulmonary disease, atrial fibrillation and who had already undergone mitral and aortic valve replacement, was admitted to hospital for congestive heart failure. Following a cycle of ultrafiltration, his clinical status improved significantly and a RHC was requested to assess cardiac hemodynamics. The 
right forearm could not be used because a peripherally inserted central catheter had been previously placed there. The left forearm was, therefore, inspected and an adequate cephalic vein was identified. The basilic vein, on the other hand, was not palpable. A 21 gauge needle was used to puncture the cephalic vein and a 0.021 inch wire was inserted through the needle. At this point, a 6 French $11 \mathrm{~cm}$ long radial introducer (Cordis Corporation, Miami Lakes, FL, USA) was passed over the wire. A 6 French Swan-Ganz catheter (Edwards Lifesciences, Irvine, CA, USA) was then inserted into the introducer but could not be advanced due to a 90 degree angle between the cephalic and subclavian veins (see Figure 1). For this reason, a 0.025 inch $150 \mathrm{~cm}$ long Emerald J-tip wire (Cordis Corporation, Miami Lakes, FL, USA) was inserted into the lumen of the catheter that measures the distal pressure. However, the wire, upon entering the subclavian vein, did not go towards the heart, despite repeated attempts, but distally towards the left arm (see Figure 2). Nevertheless, the catheter was advanced over the wire into the subclavian vein retrogradely (see Figure 3). Then the wire was removed, the balloon at the distal tip of the catheter was inflated, and the catheter was gently retracted until resistance was felt, which corresponded to the junction of the cephalic vein with the subclavian vein (see Figure 4). The catheter did not pass into the cephalic vein because the inflated balloon has a larger diameter and, at the same time, the balloon did not provoke any damage to the subclavian vein due to the flexible structure of the vascular wall. Conceptually, the situation is similar to that of a urinary catheter which is inflated to avoid displacement from the bladder. Further traction was applied so as to make the Swan-Ganz catheter as horizontal as possible. Subsequently, the wire was pushed once again and, this time, thanks to the more coaxial angle between the cephalic and subclavian veins, it was possible to reach the heart (see Figure 5 and Figure 6).

Figure 1. Venous angiography of the left cephalic vein via injection of contrast medium through the distal lumen of the Swan-Ganz catheter which unveiled an unfavorable angle that did not permit advancement of the catheter into the subclavian vein (the dotted lines depict the contour of the subclavian vein and its junction with the cephalic vein).

Figure 2. The 0.025 inch J-tip was pushed retrogradely into the subclavian vein.
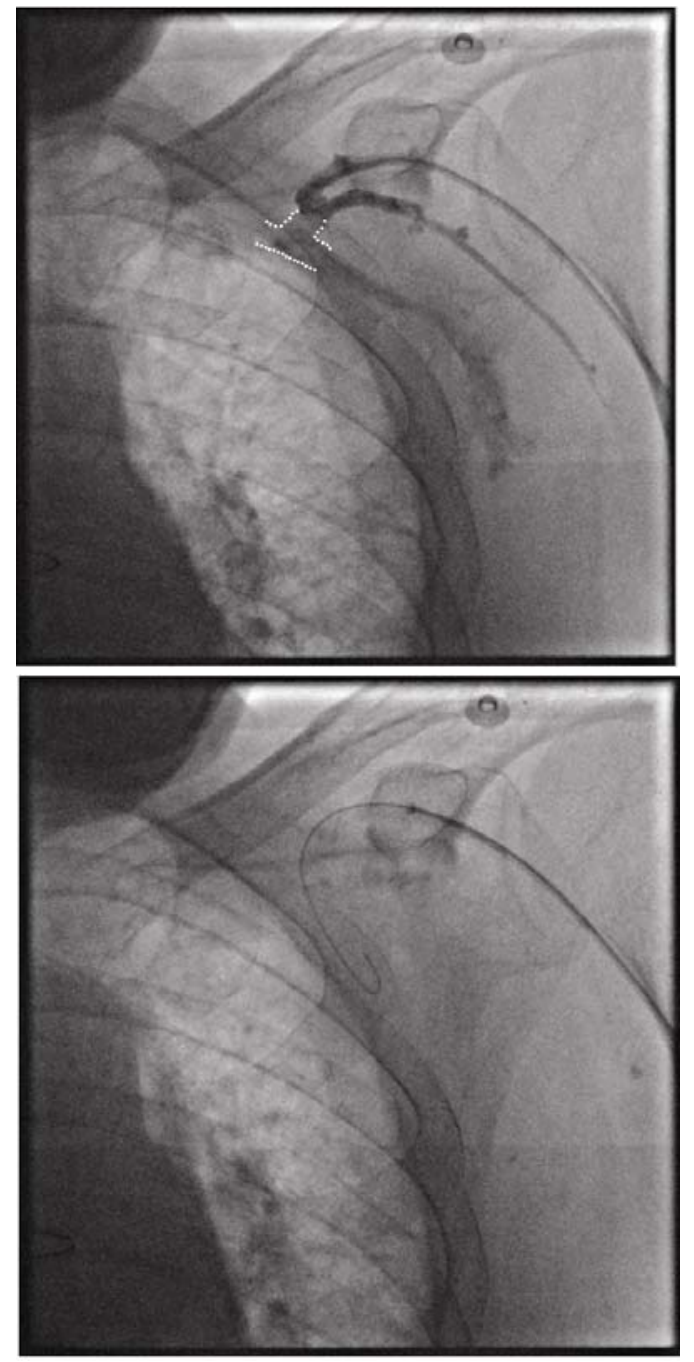


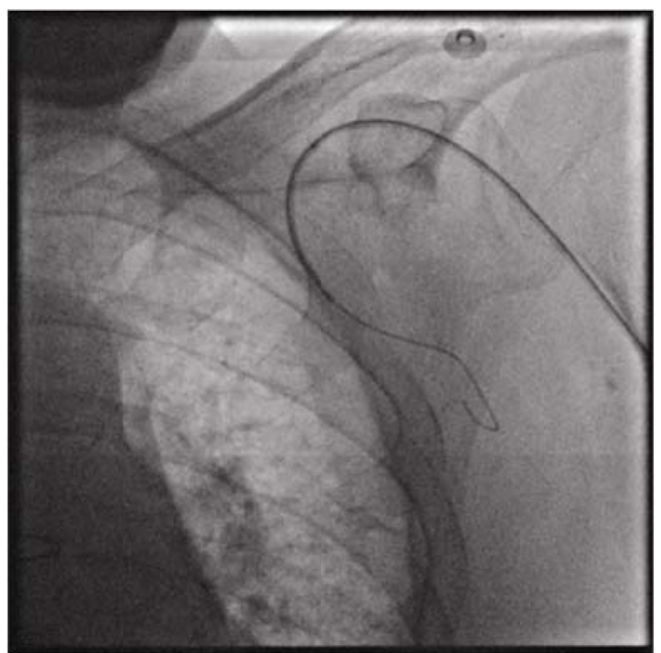

Figure 3. Thanks to the support provided by the wire, it was possible to advance the Swan-Ganz catheter into the subclavian vein, albeit in the wrong direction.

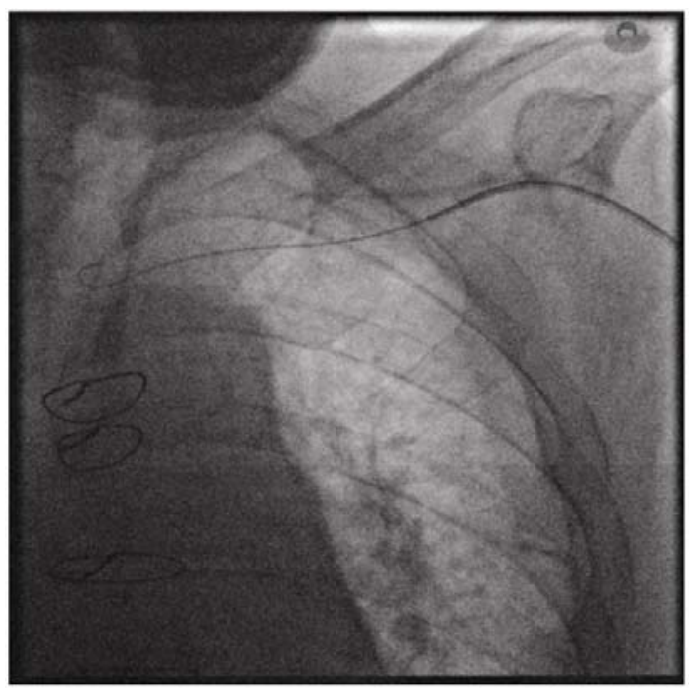

Figure 5. Further traction was applied so as to make the catheter as horizontal as possible and permit anterograde advancement of the wire into the subclavian vein.

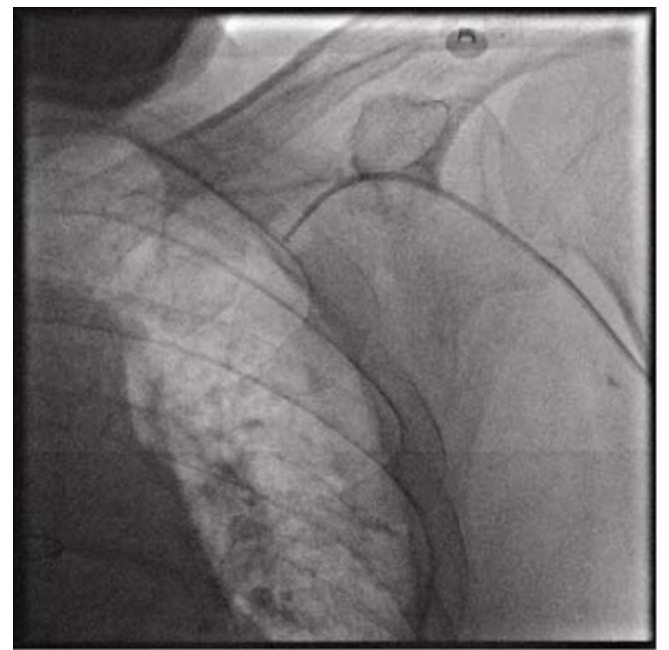

Figure 4. The wire was removed, the balloon at the distal tip of the Swan-Ganz catheter was inflated, and the catheter was retracted. Because the diameter of the balloon is greater than that of the cephalic vein, the balloon remained wedged at the junction with the subclavian vein which, in turn, was not damaged due to its flexible structure. The analogy with a urinary catheter becomes evident during this phase of the procedure.

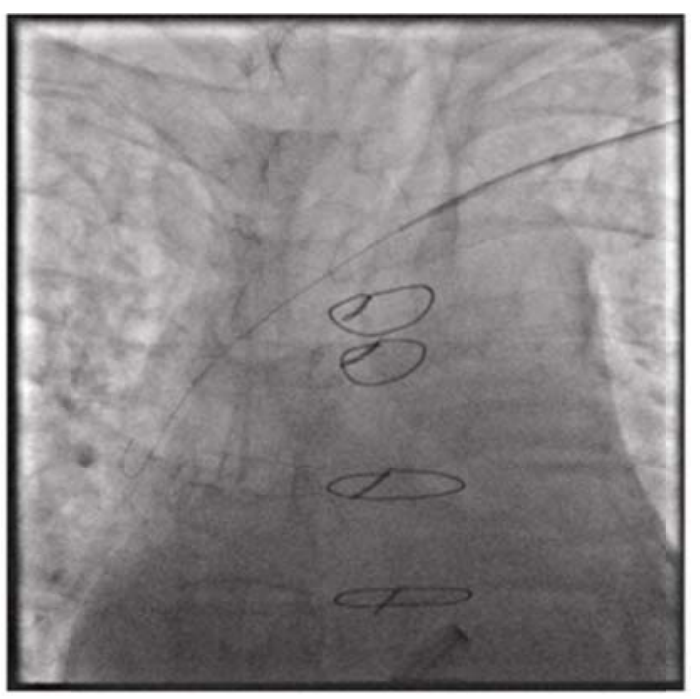

Figure 6. After deflating the balloon, the catheter and wire were advanced simultaneously and when the former reached the superior vena cava the latter was removed. At this point, the balloon was inflated once again and registration of intracardiac pressures commenced.

\section{Discussion}

RHC provides an abundance of information for the clinical cardiologist and constitutes a noble examination that every interventional cardiologist needs to master. Although usually performed via a central vein, an antecubital vein may also be accessed for the procedure. The angle between the cephalic and subclavian tends to be rather acute and, as a result, 
advancement of the Swan-Ganz catheter in this setting may be difficult. The technique described in the present report permits rapid access to the right-sided cardiac chambers via the cephalic vein. The basilic vein presents the theoretical advantage of draining directly into the subclavian vein but, compared to the cephalic vein, tends to be localized deeper in the subcutaneous tissue and, consequently, is more difficult to puncture. On the contrary, the cephalic vein is more superficial and, as a result, is easier to palpate and access. It is also the experience of the Author that the basilic vein tends to have a greater number of valves which may obstacle the advancement of the wire contained in the introducer kit. Perhaps most importantly, by using an antecubital vein the patient is not exposed to potential complications deriving from central vein puncture.

\section{References}

[1] Gilchrist IC, Kharabsheh S, Nickolaus MJ, et al. Radial approach to right heart catheterization: Early experience with a promising technique. Catheter Cardiovasc Interv. 2002; 55: 20-2. PMid:11793490 http://dx.doi.org/10.1002/ccd.10069

[2] Gilchrist IC, Moyer CD, Gascho JA. Transradial right and left heart catheterizations: A comparison to traditional femoral approach. Catheter Cardiovasc Interv. 2006; 67: 585-8. PMid:16532498 http://dx.doi.org/10.1002/ccd.20678

[3] Shah S, Boyd G, Pyne CT, et al. Right heart catheterization using antecubital venous access: Feasibility, safety and adoption rate in a tertiary center. Catheter Cardiovasc Interv. 2014; 84: 70-4. PMid:24130120 http://dx.doi.org/10.1002/ccd.25249 